\title{
Secondary Electron Yield Measurements of Carbon Nanotube Forests: Dependence on Morphology and Substrate
}

\author{
Brian Wood, Jordan Lee, Greg Wilson, T.-C. Shen, J.R. Dennison
}

\begin{abstract}
Total, secondary and backscatter electron yield data were taken with beam energies between $15 \mathrm{eV}$ and $30 \mathrm{keV}$, in conjunction with energy emission data, to determine the extent of suppression of yield caused by carbon nanotube (CNT) forest coatings on substrates. CNT forests can potentially lower substrate yield due to both its inherently low-yield carbon composition and its bundled, high aspect ratio structure. Rough surfaces, and in particular surfaces with deep high-aspect-ratio voids, can suppress yields as electrons emitted from lower lying surfaces are recaptured by surface protrusions rather than escaping the near-surface region. Yields of multilayered materials can be modeled essentially serially as a combination of the constituents. However, it is shown that suppression of yields due to CNT forest morphology is more significant than simple proportional contributions of homogeneous layered components. This effect is expected to be most pronounced at low energies, where the incident electrons interact preferentially with the CNTs.

CNT forests between 20-50 $\mu \mathrm{m}$ tall were grown on a thick silicon substrate capped with a $3 \mathrm{~nm}$ diffusion barrier of evaporated aluminum using a wet injection chemical vapor deposition (CVD) method. Yields of an annealed substrate and constituent bulk materials are also investigated. At incident electron energies above $\sim 1200 \mathrm{eV}$ the substrate secondary yield dominated those of the CNT forests, as incident electrons penetrated through the lowdensity, low-Z CNT forests and backscatter from the higher-Z substrate. At lower energies $<1200 \mathrm{eV}$, the CNT forests substantially reduced the overall yields of the substrate, and for $<500 \mathrm{eV}$ CNT forest yields were $<1$, well below the already low yields of bulk graphite. This suppressed yield at low energies is attributed to the porosity and preferred vertical alignment of the CNT forest. The yield's dependence on the height and density of the CNT forest is also discussed.
\end{abstract}

Index Terms-Electron emission, carbon nanotube forests, chemical vapor deposition, secondary electron yield, backscatter electron yield.

\section{INTRODUCTION}

$\mathrm{T}$ here is significant interest in reducing secondary electron emission from materials used for a variety of applications. This can be done by using bulk materials with intrinsically low electron yield, coating surfaces with low-yield materials [1-5], modifying the surface morphology [2,4-6], or with the use of nanocomposite material combining conducting and insulating particles to produce surface potential barriers that inhibit emission [7-9].

Multipacting issues in accelerators and waveguides, where oscillating electric fields create an avalanche effect with the electron cloud, have been mitigated with coatings, surface treatments, and use of structured nanocomposite materials $[1,2,4,6,8]$. Efficiency of traveling wave tubes (TWT) for space communicating amplifiers has also been increased with the use of textured carbon coated electrodes for the collectors [3,4,7-9].

Selection of low-Z conductors limits the incident electron interaction with bulk electrons, thereby reducing the yields [15], and is typified by use of colloidal carbon coatings such as Aquadag $^{\mathrm{TM}}$ to cover surfaces of electron optics elements and accelerator beam pipes.

Rough surfaces can also suppress yields, as electrons emitted from lower lying surfaces are recaptured by surface protrusions rather than escaping the near-surface region, as shown in Figure 1. The effect of surface roughness on electron yield has been extended to materials of high aspect ratio with deep voids; such an example are carbon velvets which tend to reduce the secondary yield of untreated planar carbon [4]. Voids in high aspect ratio materials are an extreme example of this roughness effect that act essentially as deep Faraday cups, which are very efficient at trapping electrons. Low yield CNT forest coatings might even be used to increase the effectiveness of electron collection sensors.

Modifications of yield due to CNT forest morphology are related to the angular distribution of backscattered and secondary electrons as a function of energy [10]. Analysis of emitted electron energy spectra and understanding the energy dependence of secondary yield can help separate the contributions to the yield suppression from CNT forests and other low-yield materials. While attempts to measure the secondary

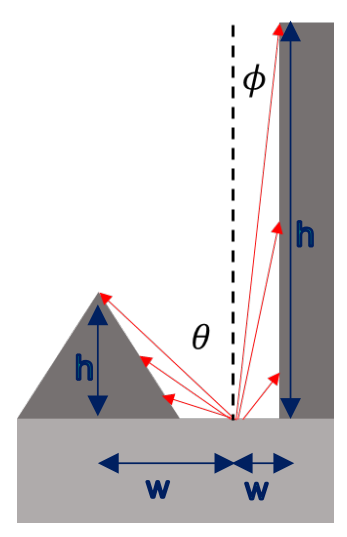

Fig. 1: Schematic illustrating how surface protrusions and high aspect ratio structures absorb emitted electrons. Low energy electrons with $\Theta>\tan ^{-1}(h / w)$ are recaptured. 
yield of individual nanotubes have been made [5], the present study focuses on the CNT forest samples as a whole, to determine the relative effects on the yield from the material composition and morphology. Forest density, height, and presence of defects are the main morphology factors that are expected to influence yield reduction of the sample. Forest density relates to the average packing density of the nanotubes which, along with CNT forest height, determines the density of bulk electrons ( $\mathrm{C}$ atoms) the incident electrons interact with, and the range that the incident electrons will penetrate into the sample.

Section II describes the growth process of CNT forests and the parameters that can be modified to produce varying height and density in forests. Characterization of CNT forests is done primarily with scanning electron microscopy (SEM). Section III briefly reviews some of the relevant aspects of electron yield production and the mechanisms that influence yields including the energy and angular distributions of secondary and backscattered electrons. Section IV outlines the experimental methods used in this study, followed by the results and conclusions of the yield measurements presented in Section V and VI.

\section{CNT FOREST GROWTH AND CHARACTERIZATION}

CNT forest samples were made in the Utah State University Nanofabrication Lab using a non-plasma enhanced wet chemical vapor deposition method [11]. Substrates of n-type silicon wafer were used with a $3+/-0.5 \mathrm{~nm}$ layer of evaporated aluminum to produce the proper in-diffusion rate of catalyst atoms. The wafer was then diced into $1 \mathrm{~cm}^{2}$ pieces and loaded into a tube furnace at $700{ }^{\circ} \mathrm{C}$. A chemical precursor of xylene with a smaller molar concentration of ferrocene was injected into the furnace, dissociating into hydrocarbons and byproducts along with iron atoms from the ferrocene. Hydrogen and argon carrier gas flowing into the furnace at $50 \mathrm{sccm}$ facilitated even distribution. Iron atoms coalesce within the substrate to form catalyst particles, allowing free carbons to dissolve into the hot Fe particles. Once saturated, rings of carbon precipitate out of the catalyst, giving a base to tip growth mechanism to produce the energetically favored tubular formation [12]. Continued precursor supply supports the vertical growth of the nanotubes. Duration of growth and precursor volume tend to determine the height of the forest, while the molar concentration of ferrocene in the precursor influences the density of the forest, with higher concentration producing denser forests, but with the possibility of more defects.

Scanning Electron Microscopy (SEM) is used to determine the height of the forest, along with its relative density and the presence of defects. Figures 2(a) and 2(b) visually illustrate the differences in density of the denser AlSi 129 sample $(0.5 \%$ ferrocene concentration) compared to the AlSi 132 sample $(0.2 \%$ ferrocene concentration). Continued growth produces inconsistent forest packing density along the height of the forest; these SEM images are taken at the base of the forests, where the density is lowest. CNT forests tend to have more pronounced vertical alignment in the intermediate region, with higher entanglement at the substrate interface and in the upper crust where CNT growth was initiated. Defects are irregularities within or on the sample, including surface

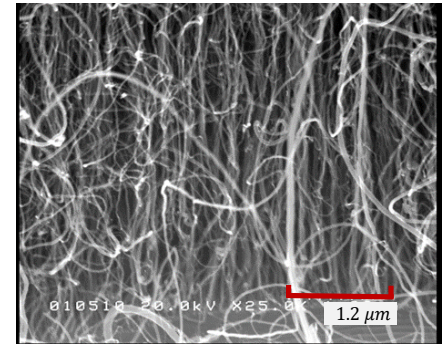

(a)

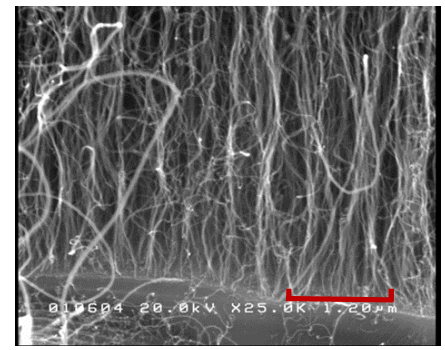

(b)

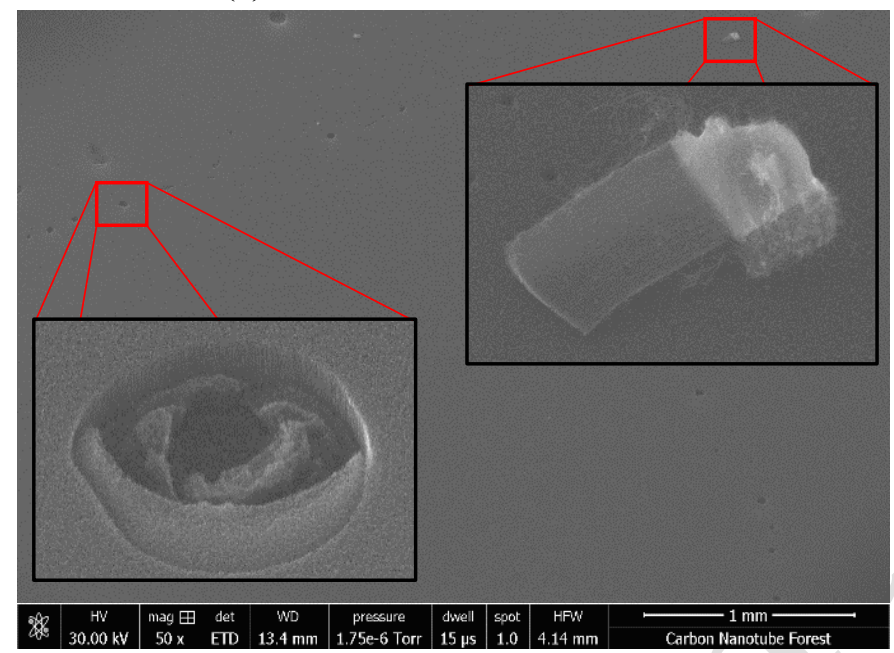

(c)

Fig. 2: Comparison of SEM images showing side-base views of the forest near the substrate interface for: (a) the denser AlSi-129 to (b) AlSi-132 samples. (c) Top view of some typical surface defects of a sample, showing (left) deformations and (right) a substrate chip that gets dislodged and pushed to the top of the forest, with nanotubes growing off its edges

TABLE I

CNT FOREST CHARACTERISTICS

\begin{tabular}{ccccc}
\hline \hline Sample & $\begin{array}{c}\text { Height } \\
(\mu \mathrm{m})\end{array}$ & $\begin{array}{c}\text { Ferrocene } \\
(\%)\end{array}$ & $\begin{array}{c}\text { Surface } \\
\text { Coverage }\end{array}$ & $\begin{array}{c}\text { Surface } \\
\text { Density } \\
\left(\mu \mathrm{g} / \mathrm{cm}^{2}\right)^{*}\end{array}$ \\
\hline AlSi 127 & $24-27$ & 0.5 & 0.90 & 150 \\
AlSi 129 & $42-51$ & 0.5 & 0.91 & 280 \\
AlSi 132 & $27-32$ & 0.2 & 0.82 & 160 \\
\hline \hline
\end{tabular}

*Assumes a $\mathrm{CN} I$ density $3 \%$ that of bulk graphite

deformation from handling or dislodged catalyst and substrate particles. Figure 2(c) shows a typical surface deformation (bottom left), along with a substrate chip that has been pushed to the surface (top right), capable of growing nanotubes along its edges. The surface has the highest density and the most overturned CNT's, an effect more pronounced for samples of higher ferrocene concentration. Samples appear to have typical defects with no major deformations aside from AlSi 132, which has portions of the sample having the forest actually scraped off, especially near the edges.

Table I lists sample heights, along with the molar ferrocene concentration during growth to distinguish the density differences. Surface coverage is also reported; this was found by counting the number of pixels above a threshold from top view photographs [13], although this is not fully indicative of 
bulk density within the forest. The bulk mass density of CNT forests grown by similar methods has been estimated as 0.02 $\mathrm{g} / \mathrm{cm}^{3}$ to $0.2 \mathrm{~g} / \mathrm{cm}^{3}$, or $1 \%$ to $9 \%$ of bulk graphite density of 2.2 $\mathrm{g} / \mathrm{cm}^{3}$. Densely packed vertically aligned nanotubes fabricated by a catalyst CVD method are reported to have mass densities on the order of $0.06 \mathrm{~g} / \mathrm{cm}^{3}$ [14]. While the wet-CVD method used for this study produces CNT's forests of less packing density, it does produce multi-walled CNT's of larger diameter, so it is reasonable to assume that the CNT densities are approximately the same. Surface density, as listed in Table I, is approximated as $3 \%$ of the bulk graphite density times, the surface coverage, times the CNT forest height.

CNT forests coatings are inhomogeneous materials with pronounced morphology. It has extreme asymmetries due to the high aspect ratio and hollow nature of the CNTs. The porosity and surface roughness of the CNT forests will also affect transport and emission. There are also many atomic and macroscopic defects. Thus, modeling the transport and emission of electrons is not straightforward. An electron can conduct preferentially along the length of the CNT, confining movement due to the orientation of the forest. Possibility of electron transfer from contacting tubes is conceivable, along with electrons emitting from the side of a tube within the forest; these may result in additional energy loss mechanisms associated with transport within the CNT forest.

\section{ELECTRON EMISSION THEORY}

Electron yield is an incident energy-dependent measure of the interactions of incident electrons with a material and characterizes the number of electrons emitted per incident electron. The total electron yield (TEY), $\sigma\left(E_{0}\right)$, is defined as the ratio emitted electron flux to the incident flux,

$\sigma\left(E_{0}\right) \equiv N_{\text {out }}^{e-} / N_{\text {in }}^{e-}=\delta\left(E_{0}\right)+\eta\left(E_{0}\right)$

It is separated into two terms, the secondary electron yield (SEY), $\delta\left(E_{0}\right)$, and backscattered electron yield (BSEY), $\eta\left(E_{0}\right)$. Figures 4 and 5 show secondary and backscattered electron yield curves.

BSEY describes electrons emitted from the material which originate from the incident beam; operationally BSE are defined as electrons with emission energies $>50 \mathrm{eV}$. Many BSE interact with the material largely through elastic (or nearlyelastic) collisions and are emitted with energies near the incident energy. Other BSE undergo one or many quasi-elastic collisions, but still escape with energies higher than most SE.

An extended three parameter empirical model has been developed to model BSEY,

$$
\begin{aligned}
& \eta_{\text {ext }}\left(E_{0}(k e V) ; \eta_{0}, \eta_{\text {peak }}, E_{\text {peak }}\right)= \\
& \mid \begin{array}{cc}
{\left[\frac{\log \left(E_{0} / 0.05\right)}{\log \left(E_{\text {peak }} / 0.05\right)}\right] \cdot\left[\left(\eta_{\text {peak }}-\eta_{0}\right) \cdot e^{\frac{-\left(E_{0}-E_{\text {peak }}\right)}{E_{\text {peak }}}}+\eta_{0}\right]} & ; 50 \mathrm{eV}<E_{0}<E_{\text {peak }} \\
{\left[\left(\eta_{\text {peak }}-\eta_{0}\right) \cdot e^{\frac{-\left(E_{0}-E_{\text {peak }}\right)}{E_{\text {peak }}}}+\eta_{0}\right]} & ; E_{o} \geq E_{\text {peak }}
\end{array}
\end{aligned}
$$

(1)

which asymptotically approaches a constant value, $\eta_{0}$, at high energies and includes the maximum BSEY yield, $\eta_{\text {peak }}$ at energy maximum yield $E_{\text {peak }}$ as adjustable parameters $[15,16]$.

SEY describes electrons emitted from the material which originate within the material and are excited through inelastic collisions with the incident electrons; operationally SE are defined as electrons with emission energies $<50 \mathrm{eV}$. Experimentally, SEY is determined by subtracting the BSEY

from the TEY. $\delta\left(E_{0}\right)$ will typically rise above unity at energy $E_{l}$, reaching its maximum yield, $\delta_{\max }$, at a specific energy, $E_{\max }$, and falling back below unity at energies above $E_{2}$. The energies $E_{1}$ and $E_{2}$ at which the yield crosses unity are called the crossover energies, where the number of emitted electrons is equal to the number of incident electrons and sample charging remains neutral. If the yield is below unity, a sample will charge negatively; if the yield $>1$, it is in a positive charging regime.

A four-parameter fit to the reduced SEY

$$
\delta\left(E_{0}\right)=\frac{\delta_{\max }}{\left[1-e^{-r_{\max }}\right]} \cdot\left(\frac{E_{0}}{E_{\max }}\right)^{1-n} \cdot\left[1-e^{\left(-r_{\max } \cdot\left(\frac{E_{0}}{E_{\max }}\right)^{n-m}\right)}\right]
$$

is used here [16,17]. Fitting parameters include $\delta_{\max }$, $E_{\text {max }}$, and two power law coefficients $n$ and $m$ related to the low energy and high energy slopes of log-log plots of SEY such as Fig. 4. $r_{\max }$ is a parameter dependent on $n$ and $m$ and fully determined by normalization of the fitting function; details of the fitting function and parameters are given in [16].

The total electron emission spectra are just the sum of the SE and BSE spectra and typically has two main peaks corresponding to SE and BSE. The shape of the distributions is very largely independent of the incident energy, which only affects the emission spectra amplitudes through the energydependent yield, $\sigma\left(E_{0}\right)=\delta\left(E_{0}\right)+\eta\left(E_{0}\right)$. A representative energy distribution of emitted electron from the CNT forests of AlSi 132, with a fit, is shown in Figure 3(a).

The SE peak rises quickly from zero emitted energy, $E$, to a peak energy at $E_{C E}=\frac{1}{3} \Phi$, usually between $1 \mathrm{eV}$ and $3 \mathrm{eV}$; it then decays more gradually back to zero at higher energies. The Chung-Everhart model [18] describes this emitted SE energy distribution, which in reduced form is [16]

$\frac{d N_{S E}\left(E_{r} \equiv E / E_{0} ; \Phi / E_{0}\right)}{d E_{r}}=\delta\left(E_{0}\right)\left\{\frac{6 \cdot\left(\Phi / E_{0}\right)^{2} \cdot\left(E_{r}\right)}{\left[E_{r}+\left(\Phi / E_{0}\right)\right]^{4}}\right\}$.

$\Phi$ is the vacuum energy surface barrier for emission. For SE to escape a material, the electron must have enough energy to cross the vacuum barrier, which is the work function for a conductor [18] or the electron affinity for dielectrics and semiconductors $[19,20,21]$. Graphite being a semi-metal has a work function of $4.86 \mathrm{eV}$ associated with it [22], and CNTs have been shown to have similar work functions of $\sim 5 \mathrm{eV}$ [23].

The BSE distribution has an upper cutoff above $E_{0}$, set by elastically scattered primary electrons, with a tail at lower energies for PE that undergo one or more lower energy inelastic collisions. The measured BSE distribution, however, is a convolution with an instrumental broadening function. This is 
typically modeled as a Gaussian with a width $\Delta E_{E}$ [16]. The reduced Gaussian model for the BSE contribution is

$$
\begin{aligned}
& \frac{d N_{B S E}\left(E_{E} \equiv E / E_{0} ; E_{0}, \Delta E_{E}\right)}{d E} \\
& =\eta\left(E_{0}\right)\left\{\left[2 \pi \cdot\left(\Delta E_{E}\right)^{2}\right]^{-1 / 2} \cdot \exp \left[\frac{1-\left(E / E_{0}\right)}{\sqrt{2}\left(\Delta E_{E} / E_{0}\right)}\right]^{2}\right\}
\end{aligned}
$$

Analysis of multilayered and composite samples is facilitated by knowledge of the electron range (the maximum distance an incident electron of specific energy will penetrate into a material). Energy is lost at an approximately constant rate (constant loss approximation) as incident electrons traverse the material; hence, an approximately uniform distribution of internal secondary electrons with depth into the material is generated. Fig. 3(b) shows the range versus incident energy calculated using a range tool developed by Wilson [24] for component bulk materials $\mathrm{Al}, \mathrm{Si}$, and graphite (nearly indistinguishable on this scale). Also shown for use with the uniform density slab model for yields is graphite scaled to $3 \%$ of bulk graphite density $\left(2.2 \mathrm{~g} / \mathrm{cm}^{3}\right)$ as a uniform density surrogate for the low-density CNT forest samples. Range is expected to scale accurately with density for bulk materials such as the $\mathrm{Si}$ substrate and $\mathrm{Al}$ barrier; however, the inhomogeneity, porosity and preferred vertical alignment of the CNT is expected to affect the electron range and electron range such that geometrical considerations have to be considered.

\section{EXPERIMENTAL SETUP}

Electron yields were measured at the USU Space Environment Effects Materials (SEEM) test facility using a custom high vacuum $\left(10^{-5} \mathrm{~Pa}\right)$ electron emission test chamber [25-27]. Two monoenergetic pulsed electron gun sources were used, a lower energy $(\sim 10 \mathrm{eV}-5000 \mathrm{eV})$, low-current $(<100$ nA) gun (Staib Instruments Model EK-5-S) and a higher energy $(5 \mathrm{keV}-30 \mathrm{keV})$, higher current $(<10 \mu \mathrm{A})$ gun (Kimball, Model EGPS-21B). Pulses used were $\sim 3-5 \mu$ s in duration at $<1 \mathrm{nA}$ $\mathrm{cm}^{-2}$ beam current densities for small beam spots $(1-2 \mathrm{~mm}$ diameter at 0.5 to $30 \mathrm{keV}$, increasing to $\sim 7 \mathrm{~mm}$ diameter at 50 $\mathrm{eV}$ and lower). In general, energies below $30 \mathrm{eV}$ may be less reliable as stray electric and magnetic fields and sample bias may alter low energy electron trajectories. Pulsed beams are implemented to reduce charging of insulators, along with a low energy $\sim 5 \mathrm{eV}$ flood gun and a $\sim 5 \mathrm{eV}$ UV LED used for a few seconds between each incident electron pulse to neutralize charge within insulating samples $[25,26]$. Energies above 5 $\mathrm{keV}$ have more variance in the pulses sent into the HGRFA, giving these measurements larger error.

Electron yields and energy spectra were measured using a fully-enclosed hemispherical grid retarding field analyzer (HGRFA) which determines absolute yield accurately $(<2 \%$ absolute uncertainty) $[25,26]$, since the encapsulating design captures almost all of the emitted electrons [25]. Concentric hemispherical grids are used both to energetically discriminate the collected electrons and to mitigate possible charging of the sample [25]. Electron pulses with varying energy impinge on the sample through the HGRFA via a drift tube. Currents traces are measured from the sample and five HGRFA detector elements, which are integrated over the pulse duration to
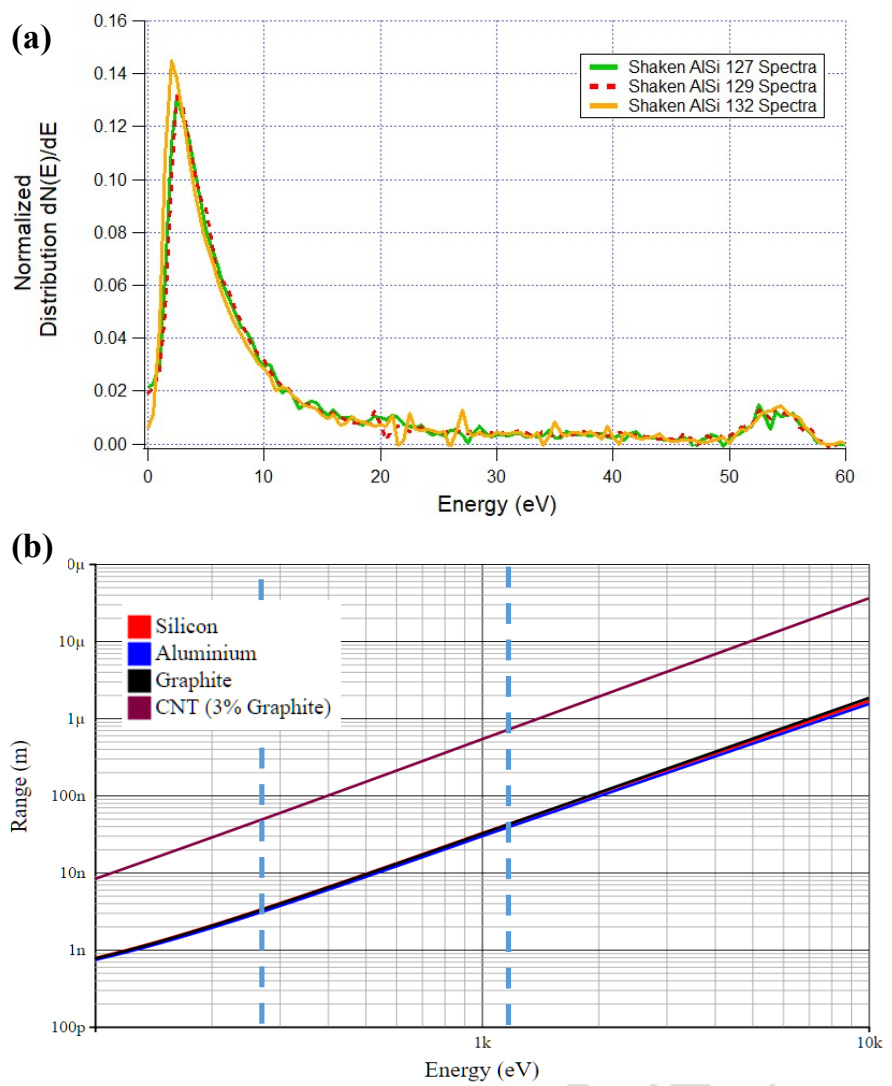

Fig. 3: (a) Emitted electron energy spectra of forest AlSi 132 with a 50 $\mathrm{eV}$ incident beam energy. (b) Electron range versus incident energy for sample materials $\mathrm{Al}$ and $\mathrm{Si}$ (indistinguishable on this scale), bulk graphite (density of $2.2 \mathrm{~g} / \mathrm{cm}^{3}$ ), and graphite scaled to $3 \%$ of bulk graphite mass density. Vertical lines indicate $270 \mathrm{eV}$ and $1200 \mathrm{eV}$ incident energies.

determine the total charge associated with the individual currents. Biasing a retarding grid to $0 \mathrm{~V}$ and $-50 \mathrm{~V}$, respectively, allows determination of total and backscattered yield calculated via Eq. (1); the difference between total and backscattered yield is the secondary electron yield. Electron emission spectra are measured by biasing this grid in $0.5 \mathrm{~V}$ increments up to the constant incident beam energy, as shown in Fig. 2(a).

\section{RESULTS}

We begin with an analysis of the yield curves of bulk Si and $\mathrm{Al}$ to the $\mathrm{Al}$ coated substrate (designated AlSi). This comparison in Figures 4(a) and 5(c) demonstrates that the yields of the coated substrate lies between the yield curves of bulk Al and $\mathrm{Si}$ and can be understood with a simple uniform slab model for yields of homogeneous multilayers [28]. From Table II, we see that bulk Al has a $\sim 20 \%$ higher maximum SEY $\delta_{\max }$ at a $\sim 20 \%$ higher energy $E_{\max }$ than bulk Si. The AlSi substrate is most similar to $\mathrm{Si}$, but consistently $\sim 8 \%$ higher in SEY (or $\sim 30 \%$ of the difference between bulk $\mathrm{Si}$ and $\mathrm{Al}$ yield curves) for $\mathrm{E}>200 \mathrm{eV}$, as is expected for a thin Al coating. Below 200 $\mathrm{eV}$ the $\mathrm{Si}, \mathrm{Al}$, and AlSi yield curves are indistinguishable within experimental uncertainties. This threshold where the AlSi yield curve departs from the $\mathrm{Al}$ yield curve at $\sim 200 \mathrm{eV}$ is consistent 
(a)

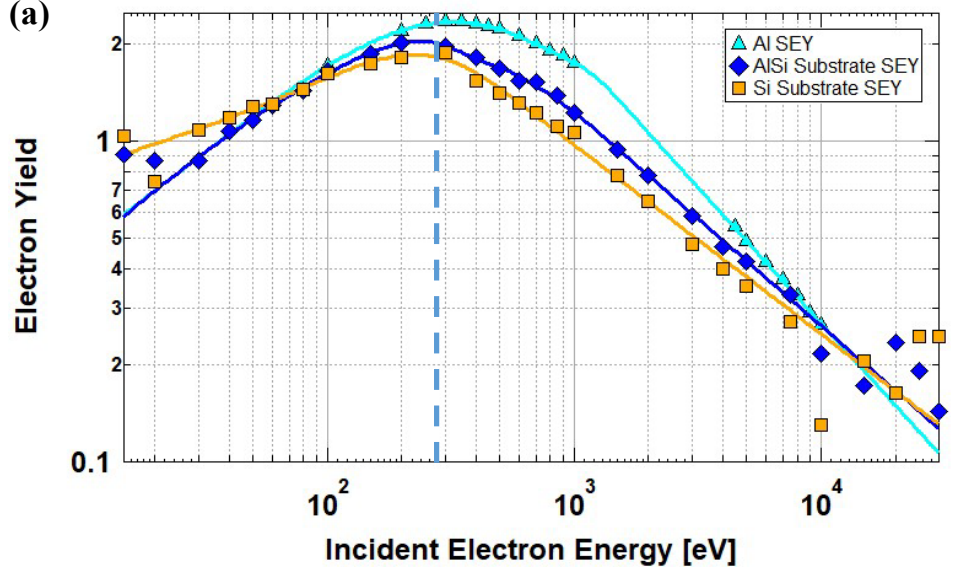

(b)

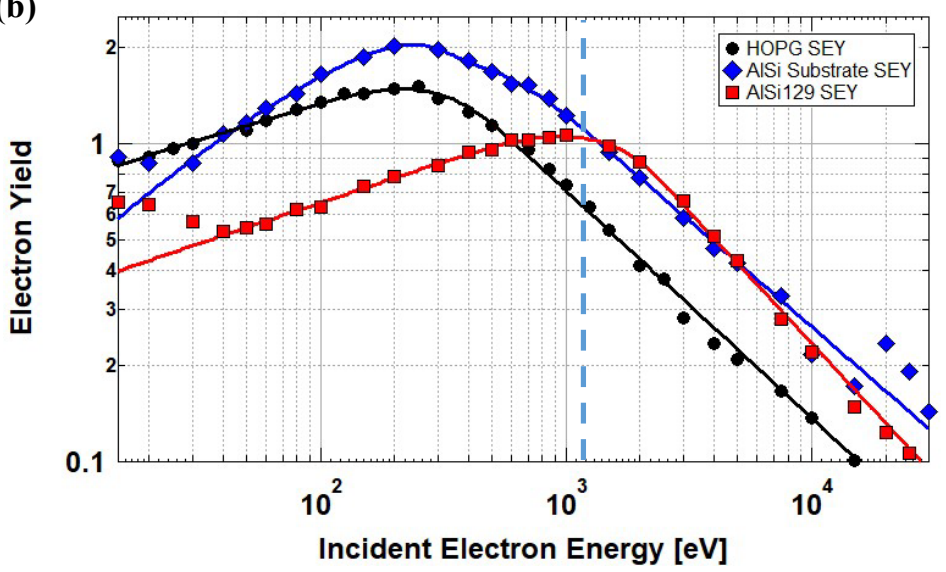

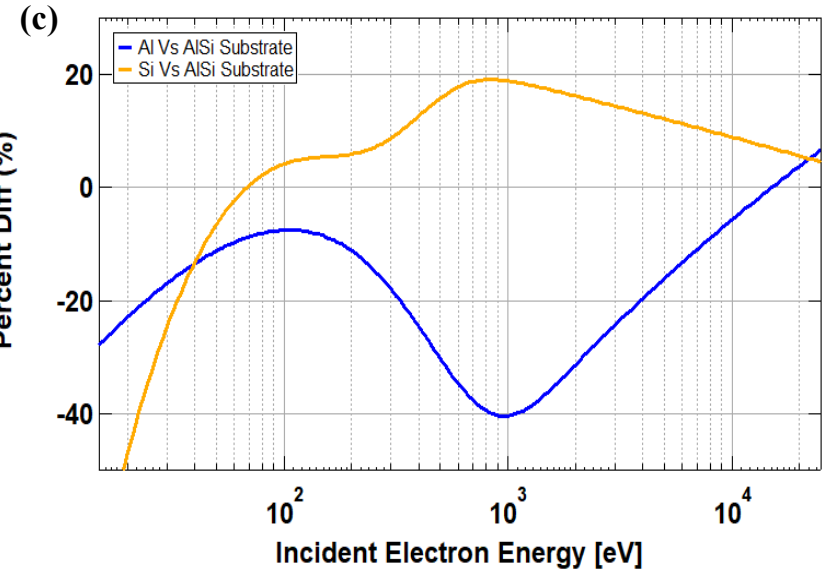

(d)

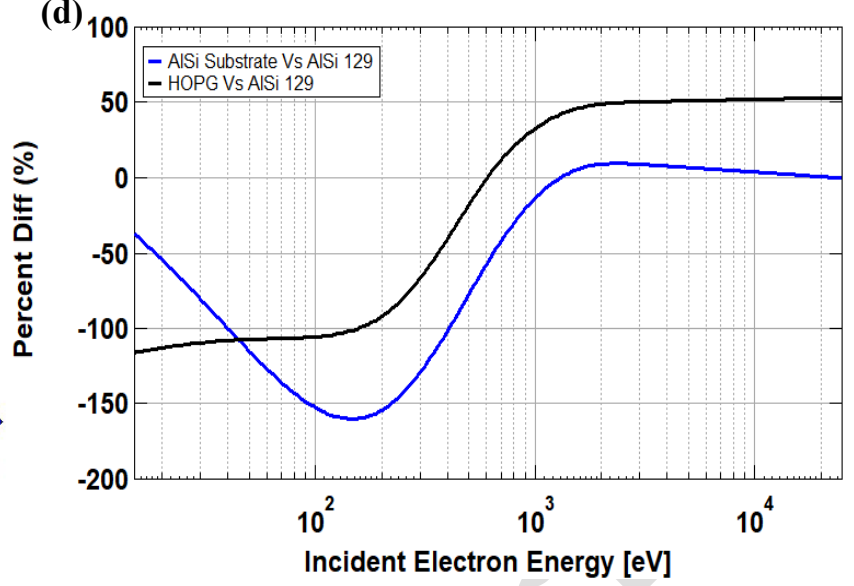

Fig. 4: Secondary electron yield measurements. (a) SEY versus incident energy of bulk Al, uncoated Si substrate, and bare Al-coated Si substrate. The vertical dashed line indicates the energy of electrons with a $3 \mathrm{~nm}$ range in bulk Al. (b) SEY versus incident energy of bulk HOPG graphite [20], a bare Al-coated Si substrate, and the AlSi 129 CNT forest sample, and fits with Eqn. (3). (c) Percent difference of Al and Si SEY from the AlSi substrate SEY versus incident energy, and (d) Percent difference of the AlSi substrate and HOPG SEY from the AlSi 129 CNT forest sample SEY. Fits in (a) and (b) are based on Eq. (3), with fitting parameters listed in Table II. Note the log-log scale for (a) and (b) and the semi-log plots for (c) and (d).

with the $3 \mathrm{~nm}$ range of a $270 \mathrm{eV}$ energy incident electron in bulk Al. The yield contributions from the AlSi substrate should be dominated by the $\mathrm{Al}$ coating below 200 to $300 \mathrm{eV}$, with the $\mathrm{Al}$ contribution falling off slightly faster than linearly at higher energies; the range increases with energy approximately as $E^{1.35}$ above $E_{\max }[24,28]$. The behavior of the AlSi substrate BSEY curve is very similar to the Si BSEY curve at all energies, as seen in Fig. 4(c).

By contrast, description of CNT forest sample yield curves as a combination of the bare AlSi substrate yield curve and a graphitic carbon yield curve becomes more difficult, as is evident in Figures 4(b) and 5(a). Figure 4(b) shows SEY versus incident energy of bulk HOPG graphite [29], a CNT-bare AlSi substrate, and the AlSi 129 CNT forest sample. Comparison of SEY of bulk HOPG to the bare AlSi substrate, shows that carbon inherently has a lower SEY, making CNTs a good candidate material for electron suppression.

Above $\sim 1200 \mathrm{eV}$, the AlSi 129 yield curve is very similar to the AlSi substrate, but about 10\% higher. The high energy SEY of the CNT forest sample is enhanced by the increased SE created by BSE from the AlSi substrate that pass back through the CNT forest on the way out; since the BSEY of AlSi is almost twice that of HOPG above $200 \mathrm{eV}$, those BSE from AlSi produce more SE.

From Figure 3(b), the range in bulk graphitic carbon is $\sim 45$ $\mathrm{nm}$ at $1200 \mathrm{eV}$, or $\sim 745 \mathrm{~nm}$ for the CNT surrogate with $\sim 3 \%$ bulk graphite density. Alternately, the energy to penetrate $\sim 35$ $\mu \mathrm{m}$ of sparse CNTs is $\sim 10 \mathrm{keV}$, a much higher energy than where the CNT forest sample yield curves begin to match the bare AlSi substrate yield curve. This suggests that the SEY suppression effect due to the CNT forest is minimal above energies about an order of magnitude less than simple uniform density slab models predict, perhaps due to the CNT morphology. The range prediction in Fig. 2 assumes a homogeneous bulk graphitic carbon material with $3 \%$ the density of graphite, while the actual samples are sparse distributions of CNT at near graphite densities with substantial vertical alignment. Morphology effects have to be considered, as the CNT forest porosity and verticality allow for easy penetration of normal incidence primary electrons, thereby reducing overall interaction with the CNTs and enhancing substrate interactions. Within the sample, primary electrons generate secondary electrons through inelastic collisions with either CNTs or the substrate, where due to their wide angular 
distribution, the forest verticality now acts as a disadvantage making reabsorption plausible.

Below $\sim 1000 \mathrm{eV}$, the AlSi 129 yield curve is much less than the bare substrate yield curve in Figure 4(b), as might be expected from a bulk HOPG graphite yield curve (with $\delta_{\max }=$ 1.34 ) that is $50 \%$ less than that of the AlSi substrate (with $\delta_{\max }$ $=2.02$ ) at $\sim 1000 \mathrm{eV}$. However, below $\sim 500 \mathrm{eV}$ the AlSi 129 yield curve is less than both the bare AlSi substrate and HOPG curves. Again, this suggests that there are substantial additional factors in reducing the CNT forest sample low-energy yields that must be attributed to the CNT morphology. At the base of the CNTs, only a small fraction of the AlSi substrate area is covered by CNT with $\mathrm{C}$ densities nearly that of graphite. If we assume rigid vertical CNT, this leads to a "patch model" for the sample where the composite yield curve is just a linear combination of HOPG and AlSi yield curves weighted by the areal coverage of the CNT at the base. Since the CNT coverage is so small, the patch model predicts the composite yield curve will be very nearly the same as (just slightly depressed due to the lower HOPG yield curve) the AlSi yield curve for all energies; this is not what is observed. We concluded that neither the uniform density slab model or the patch model are adequate to explain the measured results.

For all CNT forests samples, the largest SEY lies just above unity from $\sim 600-1500 \mathrm{eV}$, with AlSi 132 reaching the highest value of $1.16 \pm 0.02$. There are weak trends amongst the CNT forest samples at low energies with increasing $E_{\max }$, and decreasing $E_{l}$ for the AlSi 127, AlSi 129 and AlSi 132 samples, respectively (see Table II). There is also a weak trend for decreasing $\delta_{\max }$ with increasing surface density for these three sample (see Table I); such a trend is consistent in order and magnitude with increased SEY suppression scaling with the density of $\mathrm{C}$ atoms above the substrate implemented in reabsorption. The AlSi 132 yield curve in Fig. 4(b) also has some increased points between $400-700 \mathrm{eV}$; considering some of the SEM images of the AlSi 132 sample, this might be attributed to defects.

The only significant variance in the SEY amongst the CNT forests samples occurs between energies of 30-100 eV (see Figure 5(b)). AlSi 129, the tallest and denser sample, has the lowest SEY with values about $10 \%$ lower than the AlSi 132 sample in this region. AlSi 132 has a lower density than AlSi 127 , but is slightly taller on average. AlSi 132 has a lower yield from $40-150 \mathrm{eV}$, suggesting the possibility that the forest height could have more of an influence for lowering yield than the relative densities. At these energies, if you assume that most of the electron interaction is with the graphitic forest, then a denser sample will generate more secondary electrons to contribute to the yield. With forest height, chance for reabsorption of SE increases, despite the higher density of AlSi 129.

The backscatter yield curves for the CNT forest samples agree with each other to within measurement errors (see Figure 5(c)); they are also of similar magnitude to the HOPG BSEY curves above $1 \mathrm{keV}$ [29]. Below $1 \mathrm{keV}$, the sample's BSEY is lower than that of HOPG, again showing a suppressive effect at lower energies. All the CNT forest sample BSEY curves are $\sim 2.5$ times less than those of the bare AlSi substrate over the full energy range. Thus, the CNT forest coatings tend to suppress the BSEY of the substrate, regardless of their density and height. As with the SEY results, this suggests that there are
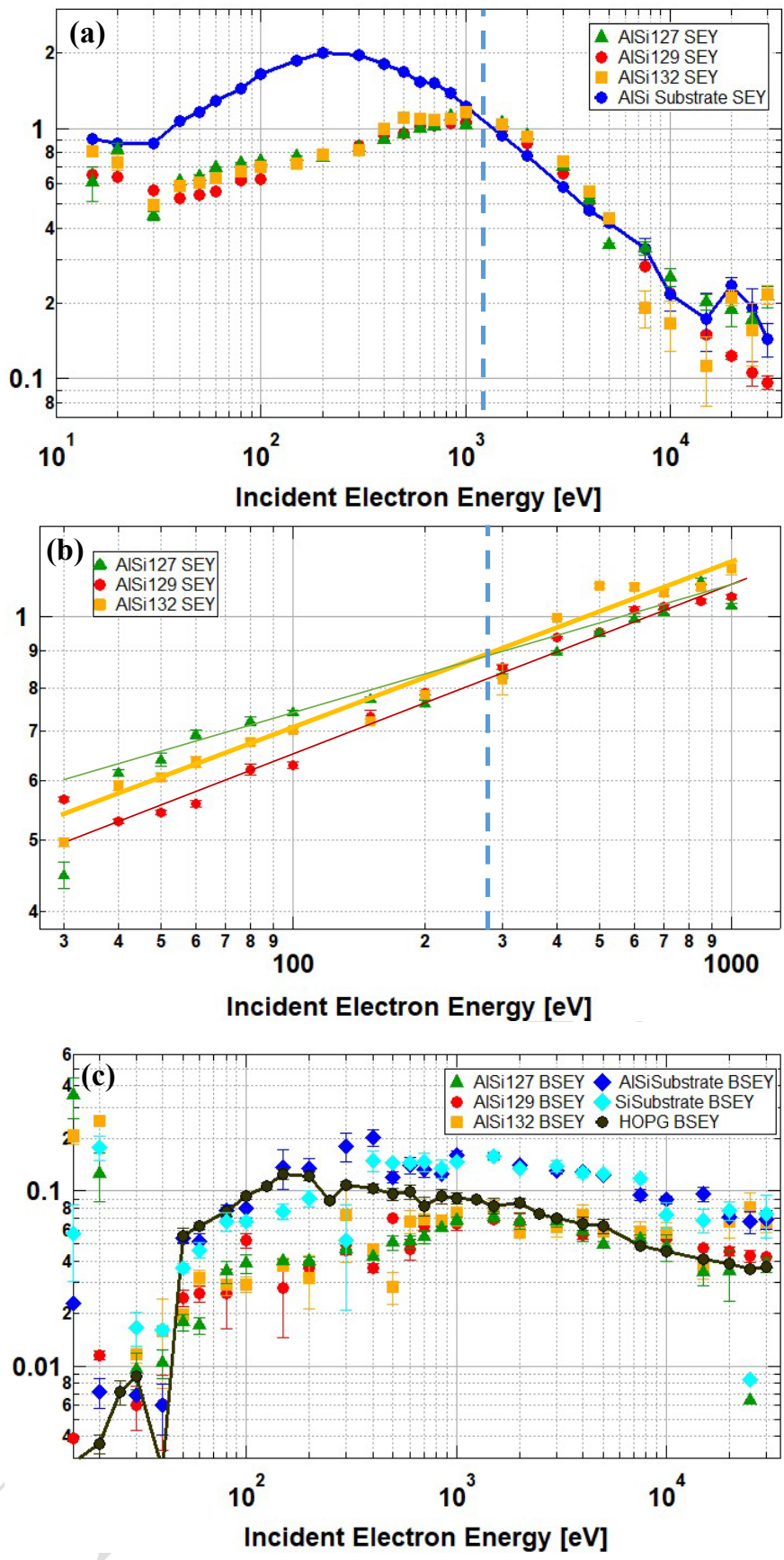

Fig. 5: Electron yield versus incident electron energy for AlSi 127, AlSi 129 and AlSi 132 CNT forest samples compared to a bare AlSi substrate. (a) SEY curve. (b) Variance in low energy SEY among the CNT forests samples. Lines are power law fits. (c) BSEY of CNT forest samples and AlSi substrate. Fits are based on Eq. (2), with fitting parameters listed in Table II.

substantial additional factors lowering the CNT forest sample low-energy yields related to the CNT morphology. Note at energies below $30 \mathrm{eV}$, the larger BSEY yields suggest that there may be some unmitigated charging effects that act to boost SE to energies above $50 \mathrm{eV}$.

Energy spectra taken on the CNT forests with a $50 \mathrm{eV}$ incident energy beam have shown no significant difference among forests. AlSi 132 has a peak amplitude of $0.15 \pm 0.01$ at 2.0 $0.3 \mathrm{eV}$, while AlSi 127 and 129 are almost identical with 
TABLE II

Electron Yield Values

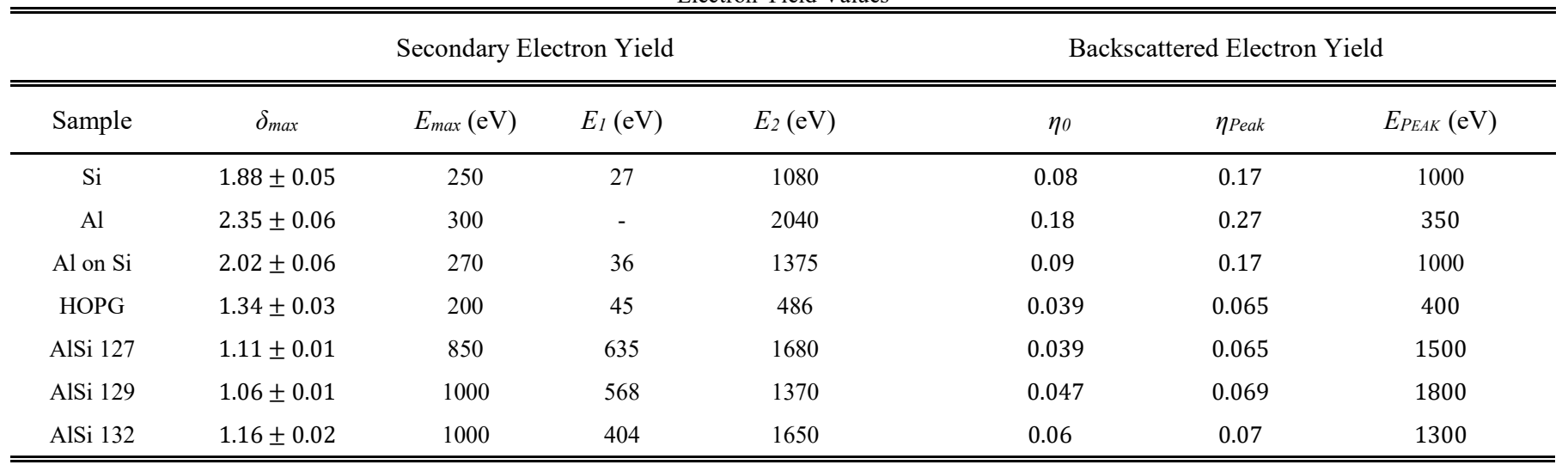

TABLE III

Secondary Electron Yield Fit Values

\begin{tabular}{ccccc}
\hline \hline Sample & $\delta_{\text {max }} \cdot\left[1-e^{\left.-r_{\text {max }}\right]^{-1}}\right.$ & $n$ & $m$ & $\beta \equiv r_{\text {max }} \cdot\left(E_{\text {max }}\right)^{n-m}$ \\
\hline Si & 97.0 & 1.66 & 0.78 & $1.60 \times 10^{-3}$ \\
Al & 322.0 & 1.76 & 0.68 & $2.93 \times 10^{-4}$ \\
Al on Si & 136.8 & 1.68 & 0.72 & $8.48 \times 10^{-4}$ \\
HOPG & 279.5 & 1.85 & 0.91 & $8.01 \times 10^{-4}$ \\
AlSi 129 & 664.1 & 1.849 & 0.925 & $1.75 \times 10^{-4}$ \\
\hline \hline
\end{tabular}

peaks of $0.13 \pm 0.01$ occurring at $2.5 \pm 0.3 \mathrm{eV}$. Backscatter peaks amplitudes and widths were all within error among all the forests.

\section{CONCLUSIONS}

Total, secondary and backscatter electron yield data taken with beam energies between $15 \mathrm{eV}$ and $30 \mathrm{keV}$ demonstrate that carbon nanotube (CNT) forest coatings on substrates substantially suppress substrate yields. This is attributed to both the inherently low yield of graphitic carbon and to the porosity and vertical alignment of the low-density CNT forest with the high aspect ratio of the CNT structures. The complex, tortuous CNT morphology has a central role in this suppression. Both SEY and BSEY of the Al-coated Si substrate are predicted well by a simple uniform density slab model. By contrast, neither the uniform density slab model nor the patch models are adequate to explain the measured results of CNT forest sample yield curves as a combination of the bare AlSi substrate and graphitic carbon yield curves.

At incident electron energies above $\sim 1200 \mathrm{eV}$ the substrate yields dominated those of the CNT forests, as incident electrons penetrated through the low-density, low-Z CNT forests and backscattered from the higher- $Z$ substrate. Above $\sim 1200 \mathrm{eV}$, the yield of the forests is $\sim 10 \%$ higher than the bare substrate, which results from more SE produced by BSE directed back out of the substrate due to a higher substrate BSEY. This $\sim 1200 \mathrm{eV}$ transition energy is about an order of magnitude lower than predicted by energy-dependent range arguments for a uniform density slab model of the composite yields. There is evidence that the transition energy will increase with increasing CNT height.
At lower energies $<1200 \mathrm{eV}$, the CNT forests substantially reduced the overall yields of the substrate, and for $<600 \mathrm{eV}$ CNT forest yields were $<1$ and well below the already low yields of bulk graphite. Although the CNT yield reduction occurs only at energies below $\sim 1200 \mathrm{eV}$, most materials' $E_{\max }$ lie below this energy, and CNT forests are therefore still effective at minimizing $\delta_{\max }$. For all CNT forests samples, the largest SEY lies just above unity in a positive charging regime from $\sim 600-1500 \mathrm{eV}$, with AlSi 132 reaching the highest value of $1.16 \pm 0.02$. The yield's dependence on the height and density of the CNT forest is a relatively small effect, but is consistent with increased influence of carbon scatter as the density and range of interaction with $\mathrm{C}$ atoms increases.

We estimate that for CNT forest heights of $\sim 150 \mu \mathrm{m}$ leading to a transition energy of $\sim 4 \mathrm{keV}$, the composite SEY will be relatively flat and $\lesssim 0.5$ over the full range of energies. Preparation of CNT forests with structure similar to those studied here, with heights of $>1 \mathrm{~mm}$, are readily prepared, suggesting that CNT forests have the potential for substantial suppression of electron yield over a broad range of energies.

\section{REFERENCES}

[1] V. Baglin, J. Bojko, O. Grobner, B. Henrist, N. Hilleret, C. Scheuerlein and M. Taborelli, "The Secondary Electron Yield of Technical Materials and its Variation with Surface Treatments" in $E P A C$, Vienna, Austria, Sep. 2000, rpt. 433.

[2] D. Ruzic, R. Moore, D. Manos, and S. Cohen, "Secondary electron yields of carbon-coated and polished stainless steel," $J$. Vacuum Sci. and Tech., vol. 20, iss. 4, 1313, 1982.

[3] A. Curren, "Carbon and carbon-coated electrodes for multistage depressed collectors for electron beam devices: a technology 
review," IEEE Trans. Electron Devices, vol. ED-33, no. 11, pp. 1902-1914, 1986.

[4] C. Jin, A. Ottaviano, and Y. Raitses. "Secondary electron emission yield from high aspect ratio carbon velvet surfaces" $J$. Appl. Phys., vol. 122, iss. 17, 173301, 2017.

[5] M. K. Alam, P. Yaghoobi, M. Chang, A. Nojeh, "Secondary Electron Yield of Multiwalled Carbon Nanotubes" (2010) Appl. Phys. Lett., vol. 97, iss. 26, 2010.

[6] R. Valizadeh, O. B. Malyshev, S. Wang, S. A. Zolotovskaya, W. A. Gillespie, and A. Abdolvand, "Low secondary yield engineered surface for electron cloud mitigation," Appl. Phys. Lett., vol. 105, 231605, 2014.

[7] L. Olano, M. E. Dávila, J. R. Dennison, and I. Montero, "Dynamic secondary electron emission in rough composite materials," submitted to J. Appl. Phys., 2018.

[8] J. d. Lara, F. Perez, M. Alfonseca, L. Galan, I. Montero, W. Roman, and D. R. Garcia-Baquero, "Multipactor prediction for on-board spacecraft RF equipment with the MEST software tool," IEEE Trans. Plasma Sci., vol. 34, no. 2, pp. 476-484, 2006.

[9] J. Wilson, E. Wintucky, K. R. Vaden, D. A. Force, I. L. Krainsky, R. N. Simons, N. R. Robbins, W. L. Menninger, D. R. Dibb, and D. E. Lewis, "Advances in space traveling-wave tubes for NASA missions," Proc. IEEE, vol. 95, no. 10, 1958-1967, Oct. 2007.

[10] N. Nickles, R. E. Davies and J. R. Dennison, "Applications of Secondary Electron Energy and Angular-Distributions to Spacecraft Charging," Proc. $6^{\text {th }}$ Spacecraft Charging Tech. Conf., Hanscom Air Force Base, MA, 2000.

[11] B.D. Wood, "Optical Characterization of Carbon Nanotube Forests," M.S. Thesis, Dept. of Physics, USU, Logan, UT, 2015.

[12] G. G. Tibbetts, "Why are carbon filaments tubular?" J. Crystal Growth, vol. 66, pp. 632-638, 1983.

[13] B.D. Wood, J.S. Dyer, V.A. Thurgood, N.A. Tomlin, J.H. Lehman, T.-C. Shen, "Optical Reflection and Absorption of Carbon Nanotube Forest Films on Substrates," J. Appl. Phys., vol. 118 , iss. 1, 2015.

[14] G.F. Zhong, T. Iwasaki, H. Kawarada, "Semi-quantitative study on the fabrication of densely packed and vertically aligned singlewalled carbon nanotubes," Carbon, vol. 44, iss. 10, 2009-2014, 2016

[15] W.Y. Chang, J.R. Dennison, J. Kite and R.E. Davies, "Effects of Evolving Surface Contamination on Spacecraft Charging," Paper AIAA-2000-0868, Proc. 38 ${ }^{\text {th }}$ AIAA Meeting on Aerospace Sci., Reno, NV, 2000.

[16] Phil Lundgreen, Justin Christensen, and JR Dennison, "Comparison of Models for Materials Parameters Used in Spacecraft Charging Codes," IEEE Trans. on Plasma Sci., 2019.

[17] A.J. Sims, "Electrostatic Charging of Spacecraft in Geosynchronous Orbit," Defence Research Agency Technical Memorandum Space 389, 1992.

[18] M.S. Chung, T.E. Everhart, "Simple Calculation of Energy Distribution of Low-Energy Secondary Electrons Emitted from Metals under Electron Bombardment," J. Appl. Phys. Vol. 45, iss. 2, 1974.

[19] E. M. Baroody, "A theory of secondary electron emission from metals," Phys. Rev. 78, 780. ,1950

[20] Quinn, J., 1962, "Range of Excited Electrons in Metals," Phys. Rev. 126, 1453.

[21] N. Balcon, D. Payan, M. Belhaj, T. Tondu, V. Inguimbert, "Secondary Electron Emission on Space Materials: Evaluation of the Total Secondary Electron Yield from Surface Potential Measurements," IEEE Trans. Plasma Sci., vol. 40, no. 2, 2012.

[22] W. N. Hansen, G. J. Hansen, Standard reference surfaces for work function measurements in air," Surf. Sci. 481, 172-184, 2001.

[23] M. Shiraishi, M. Ata, "Work Function of Carbon Nanotubes," Carbon, vol. 39, iss. 12, 1913-1917, 2001.
[24] G. Wilson, A. Starley, J. R. Dennison "Electron Range Computational Tool for Arbitrary Materials Over a Wide Energy Range," $15^{\text {th }}$ Spacecraft Charging Tech. Conf., Kobe, Japan, June 25-29, 2018.

[25] J. Christensen, "Electron Yield Measurements of High-Yield, Low Conductivity Dielectric Materials," M.S. Thesis, Dept. of Physics, USU, Logan, UT, 2017.

[26] R.C. Hoffmann, "Electron-Induced Electron Yields of Uncharged Insulating Materials," M.S. Thesis, Dept. of Physics, USU, Logan, UT, 2010.

[27] R. C. Hoffmann and J. R. Dennison, "Methods to Determine Total Electron-Induced Electron Yields Over Broad Range of Conductive \& Nonconductive Materials," IEEE Trans. Plasma Sci., vol. 40, iss. 2, 298-304, 2011.

[28] J. R. Dennison, J. C. Gillespie, A. Andersen, A. E. Jensen, J. Dekany, G. Wilson, A. M. Sim, and R. Hoffmann, "Synergistic Models of Electron Emission and Transport Measurements of Disordered $\mathrm{SiO}_{2}$," Proc. $14^{\text {th }}$ Spacecraft Charging Tech. Conf., ESA/ESTEC, Noordwijk, NI, 2016.

[29] J. R. Dennison, “Absolute Electron Emission Calibration: Round Robin Tests of Au and Graphite," $14^{\text {th }}$ Spacecraft Charging Technology Conference, ESA/ESTEC, Noordwijk, NL, 2016.

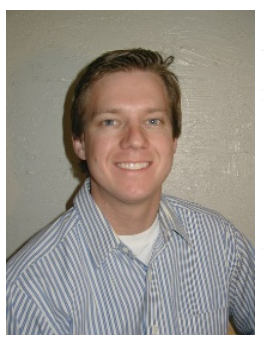

Brian Wood received his M.S. and B.S. in physics at Utah State University in Logan, UT in 2011 and 2015 respectively, where he studied nanotechnology and fabrication associated with carbon nanotubes. Currently, he has been a researcher with the Materials Physics Group for 2 years studying electron transport, electron emission, and radiation effects testing related to spacecraft charging.

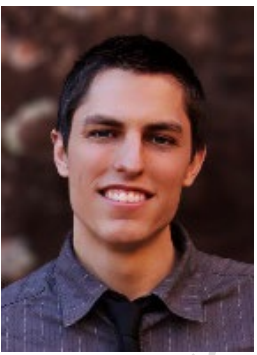

Greg Wilson received dual B.S. degrees in physics and mathematics from Utah State University in Logan, UT in 2012. He received an MS in physics from Montana State University in Bozeman, MT in 2015. He is currently a $\mathrm{PhD}$ student in physics at Utah State University. He has worked with the Materials Physics Group for four years on electron emission and luminescence studies related to spacecraft charging. He is currently working for National Technical Systems as a Senior Engineer.

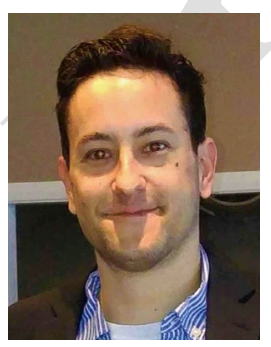

Jordan Lee received his B.S. degree in Psychology from Southern Utah University, Cedar City, UT in 2012, and a B.S. degree in Physics from Utah State University (USU), Logan, UT in 2018. Currently he is an instrumentation specialist for the Material Physics Group at USU, collecting and analyzing data relevant to electron emission and permittivity-in-for use in space-like conditions.

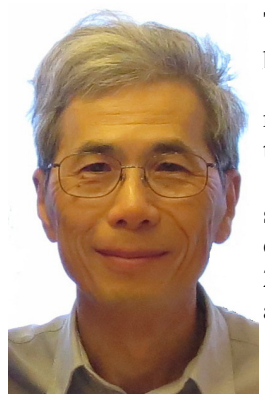

T.-C. Shen received his $\mathrm{PhD}$ degree in theoretical physics from the University of Maryland in 1985. From 1989 to 1998 he used the scanning tunneling microscope to study silicon surfaces and to pattern them at atom scales at the University of Illinois. In 1999 he joined the faculty of Utah State University and studied phosphorous $\delta$-layer in $\mathrm{Si}$ and cryogenic electronic transport of the embedded nanowires. Since 2009 he turned his research to carbon nanotube growth and applications. 


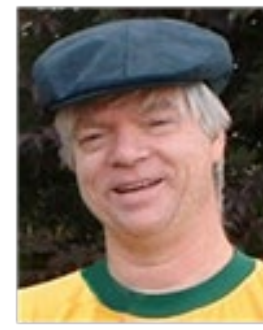

JR Dennison received the B.S. degree in physics from Appalachian State University, Boone, NC, in 1980, and the M.S. and Ph.D. degrees in physics from Virginia Tech, Blacksburg, in 1983 and 1985, respectively. He was a Research Associate with the University of Missouri-Columbia before moving to Utah State University (USU), Logan, in 1988. He is currently a Professor of physics at USU, where he leads the Materials Physics Group. He has worked in the area of electron scattering for his entire career and has focused on the electron emission and conductivity of materials related to spacecraft charging for more than two decades. IEEE member. 